\title{
Valoración médico forense del maltrato intrafamiliar a ancianos en la subdirección de Bizkaia del Instituto Vasco de Medicina Legal durante el trienio 2009-2011
}

\author{
Forensic medical examination of elder abuse in the Basque \\ Institute of Legal Medicine (Bizkaia) during 2009, 2010 and 2011
}

Resumen

Introducción: Los malos tratos a personas mayores son una realidad. Detectarlos resulta complicado y las denuncias raramente llegan de las propias víctimas. Con frecuencia, víctima y agresor comparten vínculos familiares, lo cual dificulta la denuncia. Existen pocos trabajos sobre el tema en el ámbito forense. El objetivo de este estudio es analizar sus características epidemiológicas e identificar factores asociados al maltrato en el anciano.

Material y métodos: Estudio descriptivo y retrospectivo mediante el análisis de 122 expedientes: 70 reconocidos en guardia/clínica, y 52 en UVFI. La muestra la componen víctimas de ambos sexos, de edad igual o superior a 65 años, durante el trienio 2009-2011. El estudio comprende todos los casos reconocidos en la Subdirección de Bizkaia del IVML.

Resultados: La prevalencia de reconocimientos en guardia/clínica es del 0,41\% del total de los practicados en Clínica, y del $4,3 \%$ de los practicados en la UVFI. La media de edad de las víctimas es de de 74 años. En los primeros, el agresor es el hijo (55,7\%), y en la UVFI el marido (45,7\%). Muchas de las víctimas establecen relaciones de dependencia y utilizan mecanismos de respuesta desadaptativos.

Conclusiones. Los reconocimientos practicados en guardia/clínica son inmediatos y las víctimas presentan lesiones físicas leves que no precisan de tratamiento médico. Algunas de éstas son reconocidas posteriormente en la UVFI, lo cual depende de la solicitud de los Juzgados intervinientes. Los reconocimientos practicados en UVFI son diferidos y predomina la existencia de malestar clínico en las víctimas, cuando no de un trastorno psíquico reactivo franco

Palabras clave: Prevalencia. Maltrato ancianos. Violencia doméstica. Valoración médico-forense.

\section{Abstract}

Introduction: Elder abuse is more common than people might think. Detecting is complicated because victims seldom report abuse. There is usually a family relationship between aggressor and victim. Only a few studies deal in the forensic area. The main goal of this study is to analyze and to identify the most relevant variables associated to that problem.

Materials and methods: A descriptive and retrospective study in the Basque Institute of Legal Medicine (Bizkaia), has been carried out by analyzing 122 medical records: 70 in the Forensic Clinic and 52 in the UVFI. The final sample is composed of victims of both genders older than 65 years of age. The study was conducted between 2009 and 2011 .

Results: The prevalence rate of elder abuse is $.41 \%$ among all medical records documenting some kind of violence in the Forensic Clinic, but this prevalence rate rises to $4.3 \%$ if only the records of the UVFI. Victims were women and the average age of the 74 years old. In the Forensic Clinic, the son is most often the perpetrator (55.7\%); in the UVFI the husband is most often the perpetrator $(45.7 \%)$

Conclusions: When victims are examined in the Forensic Clinic, forensic medical examination is immediate. Most of victims are suffering from light injuries, which do not require any further medical assistance. According to a court decision, some of the victims will be examined later on in the UVFI. In these cases are suffering from a significant problems and even, in some cases, from adjustment disorders.

Key words: Prevalence rate. Elder abuse. Domestic violence. Forensic medical examination.
ML. de Francisco Maíz ${ }^{1}$

MA. Solano Jaurrieta²

${ }^{1}$ Médico Forense de la Subdirección de Bizkaia del IVML. Bilbao.

${ }^{2}$ Médico Forense.

Subdirectora de la Subdirección de Bizkaia del IVML. Bilbao

Correspondencia: $M^{\mathrm{a}}$ Luisa de Francisco Maiz Servicio de Clínica MédicoForense

Buenos Aires 6, $4^{\text {a Planta }}$ 48001 Bilbao

E-mail:

francisco.ml@aju.ej-gv.es

Fecha de recepción:

9.JUL.2012

Fecha de aceptación:

12.0CT.2012 


\section{Introducción}

Los malos tratos a personas mayores son una realidad, si bien se desconoce la magnitud exacta de este fenómeno. Detectarlo resulta complicado y las denuncias raramente llegan de las propias víctimas. No es de extrañar, por tanto, que las estadísticas escaseen y su fiabilidad sea cuestionable. Con todo, los estudios revisados apuntan a que las personas mayores constituyen uno de los grupos sociales más afectados por el maltrato en sus diversas formas.

En la Primera Conferencia Nacional de Consenso sobre el anciano maltratado (Kessel H. et al. ${ }^{1}$ ), celebrada en Almería en 1995, se acuerda definir maltrato al anciano como: "Cualquier acto u omisión que produzca daño, intencionado o no, practicado sobre personas de 65 años o más, que ocurra en el medio familiar, comunitario o institucional, que vulnere 0 ponga en peligro su integridad física o psíquica, así como el principio de autonomía o el resto de los derechos fundamentales del individuo, constatable objetivamente o percibido subjetivamente".

Desde una perspectiva jurídica (CGPJ ${ }^{2,3}$ ), se podría definir el maltrato como "las acciones u omisiones, normalmente constitutivas de delito o falta, que tienen como víctima a una persona mayor de 65 años, y que se basan en una relación de proximidad o confianza, ya sea esta familiar o de otro tipo".

En lo relativo a los tipos de malos tratos a las personas mayores ${ }^{4-9}$, se pueden incluir los siguientes:

1. Maltrato físico. Se define como el uso intencionado de la fuerza física o violencia que puede dar lugar a lesión corporal, dolor físico o perjuicio. Como por ejemplo heridas, quemaduras, fracturas, uso inapropiado de fármacos, alimentación forzada o falta de ella, castigo físico, incluyendo medidas de contención (ataduras...), etc.

2. Maltrato psicológico. Supone causar intencionadamente angustia, pena, sentimiento de indignidad, miedo o aflicción por medio de actos verbales o conducta no verbal (amenazas, insultos, intimidación, humillación, ridiculización, infantilización, privación de seguridad y/o afecto, invalidación, objetificación, etc.).

3. Maltrato económico. Se define como el uso ilegal o inapropiado de fondos, propiedades y otros recursos del anciano. Incluye el obtener dinero, sin autorización o permiso, falsificación u obligación a firmar algún documento y uso inapropiado de tutela o curatela.
4. Abuso sexual. Se considera al comportamiento (gestos, insinuaciones, exhibicionismo, etc.) 0 contacto sexual de cualquier tipo, consumado o no, no consentido o con personas incapaces de dar consentimiento (violación, tocamiento, acoso sexual, hacer fotografías, etc.).

5. Negligencia. Se considera como trato negligente al rechazo, negativa o fallo para iniciar, continuar o completar cualquier parte de las obligaciones o deberes a un anciano, de forma voluntaria o involuntaria. Incluye no aportar medidas económicas o cuidados básicos como alimentación, hidratación, higiene personal, vestido, cobijo, medidas de confort, seguridad personal; incluidos en una responsabilidad implícita o acordada.

6. Abandono. Supone la deserción o abandono por un individuo que ha asumido el papel del cuidador o por la persona que tiene la custodia física. Algunos autores incluyen el abandono como una forma de negligencia del cuidador.

Cifras reales sobre el maltrato a ancianos son difíciles de obtener, por un lado debido a la negativa de la víctima a denunciar, por temor a las potenciales represalias, y por otro, porque los actos ocurren, mayoritariamente, dentro del seno familiar.

El objetivo de este estudio es analizar las características epidemiológicas de la muestra e identificar factores asociados al maltrato en ancianos.

\section{Estudio del maltrato intrafamiliar del an- ciano en el IVML: Material y métodos}

En el IVML hemos Ilevado a cabo un estudio descriptivo y retrospectivo, realizado mediante el análisis de expedientes, que en nuestra codificación hemos dividido en reconocimientos por maltrato realizados en los servicios clínicos, para los casos en los que interviene el médico forense de guardia o el médico forense generalista. En general se trata del primer contacto con la víctima y denuncian por lesiones físicas. La otra codificación corresponde a los reconocimientos por maltrato que valoramos en la Unidad de Valoración Forense Integral (UVFI). En general se trata de denuncias por maltrato habitual y la evaluación se lleva a cabo por médicos forenses expertos en psiquiatría, psicólogos y trabajadores sociales.

La valoración pericial de los casos de denuncias por malos tratos a los ancianos se lleva a cabo en los servicios clínicos de los Institutos de Medicina Legal (en nuestro caso en el Instituto Vasco de Medicina 
Legal -IVML-), según lo dispuesto en el artículo 479 de la Ley Orgánica del Poder Judicial ${ }^{10}$, donde se lleva a cabo la valoración del daño corporal y concretamente del producido como consecuencia de maltrato a personas mayores (normalmente tras la denuncia interpuesta en el Juzgado de Guardia).

La valoración pericial de las denuncias por violencia de género y por violencia doméstica se lleva a cabo en la UVFI. Estas Unidades fueron creadas a partir de la disposición adicional segunda de la Ley Orgánica $1 / 2004$ de 28 de diciembre, de medidas de protección integral contra la violencia de género ${ }^{11}$. En esta UVFI también se realizan valoraciones del maltrato intrafamiliar del anciano, ya sea hombre o mujer, sea quien sea el familiar que lo provoque.

Los criterios de inclusión de la muestra son:

- Víctimas de ambos sexos.

- Edad igual o superior a 65 años.

- Denuncia por maltrato intrafamiliar.

- Durante los años 2009, 2010 y 2011.

Hemos realizado el estudio en dos partes:

1. Análisis de expedientes por denuncias de maltrato intrafamiliar reconocidos en la guardia o en los servicios clínicos generales, a petición del Juzgado de Guardia, tras interponer la denuncia, o de Juzgados de Instrucción.

2. Análisis de expedientes por denuncias de maltrato intrafamiliar reconocidos en la UVFI. Este reconocimiento es diferido, se realiza a petición de los Juzgados de Violencia sobre la Mujer, Juzgados de Instrucción, y excepcionalmente Fiscalías.

En este estudio analizamos:

a) el número total de reconocimientos por agresión,

b) el número de reconocimientos efectuados en personas de edad igual o superior a 65 años, y

c) el número correspondiente a los reconocimientos por maltrato intrafamiliar en ancianos.

El número total de casos estudiados ha sido de 122, de los cuales 70 corresponden a reconocimientos efectuados en la guardia o en los servicios clínicos generales, y 52 a los practicados en la UVFI.
Los análisis estadísticos, las tablas, gráficas y figuras se han realizado con el programa SPSS (versión 19.00). Se han utilizado las técnicas descriptivas básicas para los datos cualitativos y cuantitativos.

\section{Resultados}

En la Tabla 1 se exponen el número total de reconocimientos en Clínica realizados durante los años 2009, 2010 y 2011. Observamos que en el año 2011 se incrementaron los reconocimientos un $22 \%$, respecto al año anterior. Del total de agresiones, el $2,68 \%$ se realizaron en personas de edad igual o superior a 65 años, de las cuales un 15,38\% fueron maltrato intrafamiliar. Vemos como en el año 2009 la proporción de maltrato intrafamiliar es más elevada, un tercio más de las ocurridas en los años 2010 y 2011.

De los reconocimientos efectuados en la UVFI durante el trienio 2009-2011, un 4,3\% corresponde a personas mayores de 65 años. El porcentaje mayor corresponde al año 2010.

En la misma tabla observamos que sólo el 8,57\% de víctimas reconocidas inicialmente por maltrato en Clínica fueron posteriormente valoradas en la UVFI por el equipo multidisciplinar.

\section{Resultados estadísticos de los reconocimientos en guardia y en servicios clínicos generales por maltrato intrafamiliar en mayores de 65 años}

Los resultados del estudio estadístico durante los años 2009, 2010 y 2011, tras el análisis de 70 expedientes $(\mathrm{N}=70)$, son los siguientes: 
Del total de seis partidos judiciales que componen la jurisdicción de Bizkaia, y reconocidos en los servicios clínicos de la subdirección de Bizkaia del IVML, un $70 \%$ pertenecen al partido judicial de Bilbao, el 18,6\% al de Barakaldo y el resto a los demás partidos judiciales de la provincia.

Dos tercios de los reconocimientos practicados se efectuaron en mujeres $(65,71 \%)$, con una edad media de 74,83 años. El estado civil no consta en la mayoría de los expedientes (85\%). En los que consta, el $80 \%$ están casados y el $20 \%$ son viudos.

El $81,2 \%$ de las agresiones intrafamiliares ocurren en el domicilio, el resto acontece en otros lugares, en general, cercanos al domicilio: escalera, local, calle, etc. En más de la mitad de los casos $(55,7 \%)$ el agresor es hijo de la víctima, en el $15,7 \%$ es la pareja, y en el $28,6 \%$ el maltrato lo propician otros familiares (hermano, nuera, yerno, sobrino, nieto, etc.).

En el $83,8 \%$ las agresiones se producen mediante manos y pies, siendo la utilización de objetos contundentes poco frecuente $(8,8 \%)$.

En la codificación de las lesiones físicas hemos considerado como leves aquellas que tardan en curar menos de 15 días, como menos graves cuando la curación se produce entre los 15 y los 30 días, y lesiones graves cuando el tiempo de curación es superior a 30 días.

La Figura 1 muestra los resultados. Como vemos no se objetivaron lesiones físicas en el $10 \%$ de los reconocimientos practicados. En el $84 \%$ de los casos las lesiones fueron de carácter leve, repartiéndose el $6 \%$ restante entre las lesiones menos graves y graves, en porcentajes similares.

En la muestra analizada el tiempo de curación medio (en días) es de 8,78 días, la mediana de 6 días.

En la Figura 2 se muestran los tiempos de curación y sus frecuencias. Observamos la dispersión de un caso de lesiones grave, con 115 días de curación.

\section{Figura 1.}

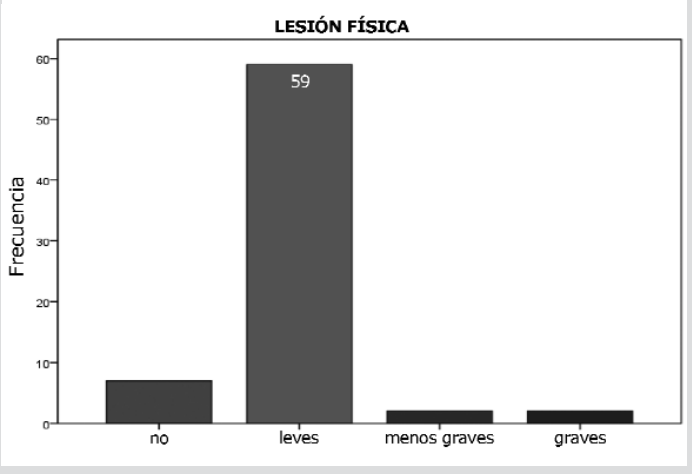

El $80,9 \%$ de la muestra solicitó asistencia médica tras la agresión, el 16,2\% no la solicitó, y un 2,9\% precisó tratamiento médico para su curación. El $85 \%$ de las víctimas de la muestra analizada no presenta secuelas físicas.

\section{Resultados estadísticos de los reconocimientos efectuados en la UVFI por maltrato intrafami- liar en mayores de 65 años}

La muestra se compone de 52 expedientes: 16 del año 2009, 20 casos del año 2010, y 16 del año 2011.

En la mitad de los casos la valoración es solicitada por órganos judiciales de Bilbao. Las valoraciones efectuadas para los Juzgados de Barakaldo suponen el $37,5 \%$ de los reconocimientos. El resto $(12,5 \%)$ fueron solicitados por los juzgados de Getxo, Gernika y Balmaseda.

El $87,50 \%$ de la muestra está compuesta por mujeres. La edad media de la muestra es de 73,71 años. La Figura 3 muestra la distribución de frecuencias por edad. El 47,92\% se encuentran en el rango de edad 65-70, y un $20,83 \%$ entre los $81-85$ años.

Más de la mitad de las víctimas están casadas (61\%), el $22 \%$ separadas o divorciados, y el $17,1 \%$ viudas. Todos los reconocimientos practicados en la UVFI a personas de edad igual o superior a 65 años, corresponden a víctimas de nacionalidad española. El $85 \%$ de las víctimas tienen estudios primarios, un $10 \%$ no tiene estudios y un $5 \%$ tiene el graduado.

En el $45,7 \%$ de los casos el agresor es el marido, en el $37 \%$ el hijo, en el $13 \%$ la ex pareja. Sólo en dos casos de la muestra analizada $(4,2 \%)$ la agresora fue la mujer.

De las víctimas citadas para reconocimiento en la UVFI un $19,1 \%$ no acudió, y el $8,5 \%$ lo hizo de forma incompleta. En el $72,3 \%$ se practicó el reconocimiento completo. El 95,5\% de la muestra analizada no tenía antecedentes en la UVFI (no había sido reconocida previamente).

En el $97,6 \%$ de los casos de la muestra, las víctimas refieren la existencia de maltrato repetido.

En la Figura 4 observamos que el tipo de maltrato más frecuente es el psicológico y físico asociado, que se produce en el $66,7 \%$ de la muestra analizada. Le sigue en frecuencia el maltrato psicológico con un $21,4 \%$. En nuestro estudio solo tenemos un caso de abuso sexual. No nos han solicitado ningún caso para valoración de maltrato económico, negligencia o abandono del anciano. 
El $78 \%$ de las víctimas reconocidas no había efectuado denuncias con anterioridad, el 17,1\% lo había hecho en una ocasión, y un 4,9\% en más de una ocasión.

El 3\% de la muestra reconoció haber tenido parejas violentas previas y un $10 \%$ de víctimas reconocieron haber retirado la denuncia.

Se interpuso orden de protección en el 53,3\% de los casos valorados, la cual se respetó en el 90,5\% de los casos.

Un $82,5 \%$ de las víctimas reconocidas en la UVFI niegan haber sufrido lesiones físicas en el momento del reconocimiento. El 10\% refiere haber sufrido lesiones leves, y el 7,5\% lesiones de entre 15 y 30 días de curación (menos graves).

Antecedentes psiquiátricos: Más de la mitad de los casos $(56,1 \%)$ no presenta patología previa. El $16,7 \%$ de la muestra padece un trastorno afectivo. La misma proporción presenta una discapacidad intelectual en forma de deterioro cognitivo, tipo enfermedad de Alzheimer. El diagnóstico de trastorno de personalidad y de psicosis lo tiene el $4,2 \%$ de la muestra, para cada trastorno.

Trastornos reactivos: En la Tabla 2 observamos que el trastorno más frecuente hallado en las víctimas mayores de 65 años reconocidas en la UVFI es el Trastorno Adaptativo con síntomas de depresión prevalentes, seguido por el Trastorno Depresivo Mayor.

No hemos detectado ningún caso de Trastorno por Estrés Postraumático, ni de Trastorno Disociativo, ni de Cambio de Personalidad, los cuales estaban codificados en el presente estudio.

Tratamiento: En la Figura 5 observamos que el $74,4 \%$ de la muestra no recibe tratamiento en el momento que se está llevando a cabo la valoración, y/o no lo ha precisado como consecuencia de los hechos que motivaron la denuncia. El 11,6\% recibe tratamiento por su Médico de Atención Primaria y la misma proporción tratamiento psiquiátrico. El 2,3\% precisa tratamiento combinado.

Tipos de relación: La Tabla 3 muestra los porcentajes según el tipo de relación prevalente. Observamos que casi la mitad de la muestra reconoce mantener un patrón de relación de dependencia.

Tipos de respuesta de la víctima: En la valoración de víctimas por denuncias de maltrato, efectuada en la UVFI, vemos que existe un ajuste inadecuado, por utilización de mecanismos de defensa desadaptativos, en el $41,9 \%$ de los casos. El estilo cognitivo más frecuente es la habituación (38,2\%), seguido

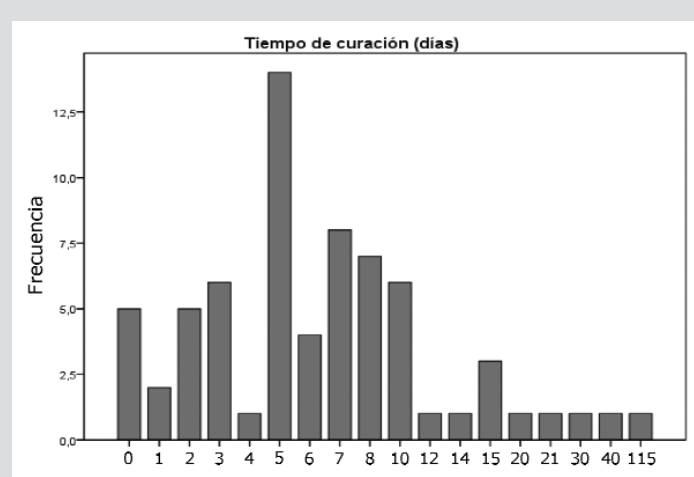

Figura 2.

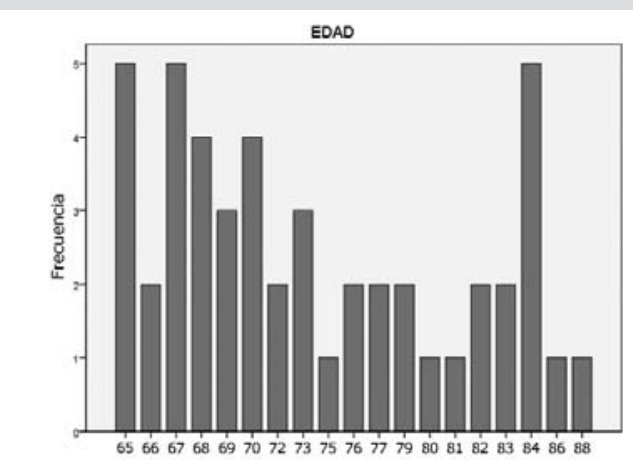

Figura 3.

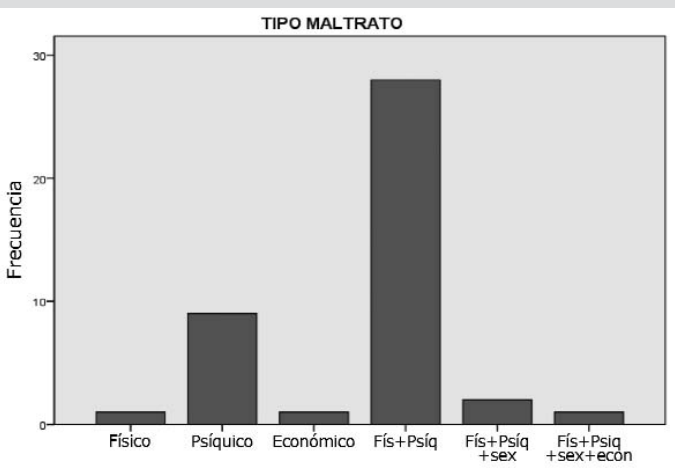

Figura 4.

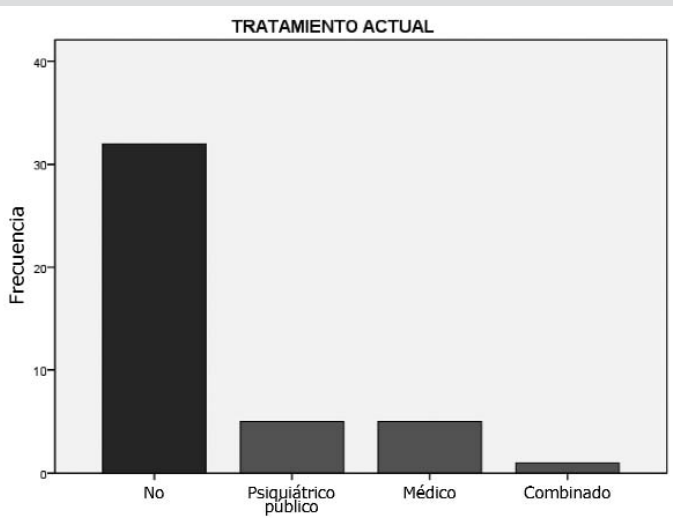

Figura 5. 


\begin{tabular}{|c|c|c|c|}
\hline \multirow{6}{*}{$\begin{array}{r}\text { Tabla } 2 . \\
\text { Lesiones psíquicas. }\end{array}$} & Tipo de trastorno & Frecuencia & Porcentaje \\
\hline & Adaptativo mixto & 4 & 8,3 \\
\hline & Adaptativo depresivo & 9 & 18,8 \\
\hline & Adaptativo ansiedad & 4 & 8,3 \\
\hline & Depresivo mayor & 5 & 10,4 \\
\hline & No existe / No diagnosticado / Otro & 26 & 54,2 \\
\hline \multirow{6}{*}{$\begin{array}{l}\text { Tabla } 3 . \\
\text { Tipos de relación. }\end{array}$} & Tipos de relación & Frecuencia & Porcentaje \\
\hline & Dependencia & 17 & 48,6 \\
\hline & Sumisión & 6 & 17,1 \\
\hline & Influencia & 5 & 14,3 \\
\hline & Depresivo & 11 & 34,4 \\
\hline & Otros & 1 & 3,2 \\
\hline
\end{tabular}

por la racionalización $(34,3 \%)$ y por la minimización $(28,6 \%)$ del maltrato. Las respuestas de autoinculpación, confusión y negación son menos frecuentes (13,3\%, 10\%, y 8,6\%, respectivamente).

El $41,4 \%$ de la muestra refiere presentar malestar clínico. Un 48\% no tiene una percepción ajustada del riesgo. El 96,4\% niega ideación suicida. El $35,7 \%$ presenta estresores cotidianos añadidos.

\section{Discusión}

No conocemos estudios sobre el maltrato intrafamiliar a personas mayores en el ámbito legal con los que podamos comparar los resultados obtenidos en nuestra investigación. Las cifras obtenidas en los estudios de prevalencia realizados en otros ámbitos, probablemente sean superiores a las obtenidas en nuestro estudio, debido a que pocos casos llegan a la denuncia.

La prevalencia del maltrato y la negligencia hacia personas mayores es muy difícil de estimar ${ }^{12,13}$. Muchos casos continúan sin ser reconocidos o informados por el aislamiento en el que se encuentran las posibles víctimas ${ }^{14,15}$. Además estas situaciones ocurren, mayoritariamente, dentro del seno familiar (Gaioli CC, Rodríguez $\mathrm{RA}^{7}$ ) y esto hace que sea más difícil detectar los casos de maltrato. Finalmente, el uso de metodologías distintas en los estudios realizados dificulta comparar los resultados obtenidos.
Un reciente estudio de prevalencia de malos tratos a personas mayores efectuado en la C.A. del País $V_{\text {Vasco }}{ }^{16}$ analiza la incidencia de malos tratos hacia las personas mayores, tanto en el ámbito nacional como en el internacional. Encuestas nacionales muestran una gran similitud con una prevalencia de alrededor del 5\%. Así Teresa Bazo ${ }^{17}$ encuentra una prevalencia del $4,7 \%$, siendo las mujeres las víctimas más frecuentes de este tipo de violencia y los hijos/as aparecen como los responsables de malos tratos más frecuentes. Resultados equiparables a los obtenidos en nuestro estudio. Estudios internacionales ${ }^{18-20}$ hallan una prevalencia del $6,3 \%$, siendo los malos tratos psicológicos el tipo más frecuente, seguido de los malos tratos económicos, negligencia y físicos. En nuestro estudio es más frecuente el maltrato psicológico y físico asociado $(66,7 \%)$. Numerosos estudios ${ }^{20-24}$ concluyen que es en el ámbito doméstico donde se producen los malos tratos hacia las personas mayores con mayor frecuencia.

Los resultados de sospecha de prevalencia ${ }^{25-28}$ de maltrato arrojan prevalencias mucho mayores, de entre el $12,1 \%$ al 52,6\%. Así Pérez-Rojo et al. ${ }^{25}$ encontraron una prevalencia del $12,1 \%$, siendo el tipo más frecuente de sospecha el psicológico, seguido por la presencia simultánea de diferentes tipos, especialmente los malos tratos psicológicos, físicos y sexuales.

\section{Conclusiones del maltrato intrafamiliar a ancianos}

\section{En los reconocimientos practicados en la guar- dia y en los servicios clínicos generales}

Existe un incremento de los reconocimientos por agresión en el último año del trienio analizado (2011), sin embargo el porcentaje de reconocimientos por maltrato a ancianos es equiparable al de los años anteriores. En el año 2009 el porcentaje de reconocimientos por maltrato intrafamiliar fue casi el doble $(22,83 \%)$ que en los años 2010 y 2011 $(12,74$ y $12,28 \%$, respectivamente).

Las víctimas son mayoritariamente mujeres $(65,71 \%)$ con una edad media de 74,83 años. Las agresiones ocurren en el domicilio, el agresor es el hijo en más de la mitad de los casos. Las agresiones se producen mediante manos y/o pies con resultado de lesiones leves en la mayoría de los casos (84\%). Un $10 \%$ no presenta lesiones objetivables. El tiempo de curación medio es de 8,78 días. La mayoría de las víctimas solicitó asistencia facultativa $(80,9 \%)$ y un $2,9 \%$ precisó, además, tratamiento médico para su curación. La mayoría de las víctimas (85\%) no presenta secuelas físicas. 


\section{En los reconocimientos practicados en la UVFI}

Los reconocimientos por maltrato intrafamiliar a ancianos suponen el $4,3 \%$ de los reconocimientos practicados en la UVFI. EI mayor porcentaje corresponde al año 2010 (5,48\%). Destaca el absentismo, ya que un $19 \%$ de las víctimas no acuden a los reconocimientos concertados, y el 8,5\% lo hace de forma incompleta. La mayoría de las víctimas son mujeres $(87,5 \%)$, con una edad media de 73,71 años. Más de la mitad están casadas (61\%). En la mayoría de los casos el agresor es el marido $(45,7 \%)$ o el hijo (37\%). Las mayoría de las víctimas refieren maltrato habitual $(97,6 \%)$. El $22 \%$ había efectuado denuncias con anterioridad. En la mitad de los casos se interpuso medida de protección (53,3\%), la cual se respetó en la mayoría de los casos (90,5\%). La mayoría de las víctimas niegan haber sufrido lesiones físicas (82,5\%), cuando existen son de carácter leve. El tipo de maltrato más frecuente es el psicológico y físico asociado (66,7\%), seguido por el psicológico $(21,4 \%)$, el maltrato económico y abuso sexual son poco frecuentes, no hemos valorado ningún caso de abandono al anciano o negligencia. Respecto a las lesiones psíquicas, casi la mitad de la muestra refiere presentar un malestar clínico reactivo a su situación vivencial, no tienen una percepción ajustada del riesgo y tienen estresores cotidianos asociados. El trastorno más frecuente hallado es el trastorno adaptativo con síntomas de depresión prevalentes, seguido por el trastorno depresivo mayor. La mayoría de las víctimas no presenta antecedentes psiquiátricos previos (56\%), entre los que los presentan predomina el trastorno afectivo y el deterioro cognitivo. La mayoría de la muestra no recibe tratamiento médico $(74,4 \%)$. El tipo de relación más prevalente entre víctima y agresor es el de dependencia y los tipos de respuesta más habituales en la víctima son los de habituación, racionalización y minimización.

\section{Las diferencias halladas en la comparativa reco- nocimientos practicados en Clínica/UVFI, en las variables comunes analizadas son las siguientes}

1. La valoración Médico Legal del maltrato en guardia o en los servicios clínicos generales es normalmente inmediata a la denuncia, y transversal. Se lleva a cabo en la Clínica Médico Forense por un médico-forense generalista. La valoración por maltrato realizada en la UVFI es diferida, retrospectiva y longitudinal, y se realiza por un equipo multidisciplinar: trabajador social, psicólogo y médico forense experto en psiquiatría.

2. El partido judicial de Bilbao solicita porcentualmente más reconocimientos durante la guardia. El partido judicial de Barakaldo solicita más valoraciones a la UVFI.

3. La proporción de mujeres es mayor en los reconocimientos en la UVFI: $87,50 \%$, en tanto que en los reconocimientos en Clínica suponen el $65,71 \%$.

4. La edad media es similar (74,83 / 73,71 años). La distribución de la edad es regular en los reconocimientos en Clínica, distribuyéndose de forma irregular en los reconocimientos en la UVFI (el $50 \%$ de los valorados tienen entre 65-70 años).

5. El agresor más frecuente en las valoraciones en Clínica es el hijo (50\%), en tanto que en las realizadas en la UVFI es el marido (45,7\% o ex pareja $13 \%$ ).

6. En los reconocimientos efectuados en la guardia y en los servicios clínicos predominan las lesiones físicas (90\%), siendo inexistentes en la mayoría de los reconocimientos en la UVFI (82,5\%).

7. Las lesiones psíquicas no se valoran en el reconocimiento inicial en la guardia, en tanto que su valoración es fundamental en las valoraciones efectuadas en la UVFI.

Como reflexión final destacamos la elevada vulnerabilidad de los ancianos, que en no pocas ocasiones son objeto de maltrato activo: físico, psicológico, económico, sexual, o en forma de negligencia o abandono, y en otras, que desconocemos porque no han sido objeto de denuncia. De ahí la importancia de la intervención de los agentes sanitarios y sociales que deben poner en marcha todos los mecanismos a su alcance para la prevención del maltrato en ancianos ante la sospecha de situaciones de riesgo.

\section{Agradecimientos}

A nuestros compañeros de la Subdirección de Bizkaia del IVML y a los componentes de la UVFI ya que han contribuido con su trabajo en la recogida de los datos utilizados en nuestro estudio. 


\section{Bibliografía}

1. Kessel Sardiñas H, Marín Gámez N, Maturana Navarrete N, Castañeda Fábrega I, Pageo Giménez MM, Larrión Zugasti JL. 1996. Primera Conferencia Nacional de Consenso sobre el anciano maltratado. Rev Esp Geriatr Gerontol. 1996;31 (6):367-72.

2. Consejo General del Poder Judicial (CGPJ). Foro de Justicia y Discapacidad. Personas Mayores Vulnerables: Maltrato y Abuso, 2009.

3. CGPJ. Observatorio contra la Violencia doméstica y de género del Consejo General del Poder Judicial [citado 30 Jun 2010]. Disponible en: www.poderjudicial.es/eversuite/GetRecords?Template =cgpj/cgpj/ principal.htm.

4. Lachs MS, Pillemer K. Elder Abuse. Lancet 2004,364:1263-73

5. Lachs MS, Pillemer K. Current concepts: Abuse and neglect of elderly persons. N Engl J Med. 1995;332(7):437-43.

6. Muñoz J. Personas mayores y malos tratos. Madrid: Ediciones Pirámide, 2004.

7. Sánchez del Corral F, Ruipérez I. "Violencia en el anciano". Madrid 2004, Portal Mayores, Informes Portal Mayores, $\mathrm{n}^{\circ}$ 21. Disponible en: http://imsersomayores.csic.es/documentos/documentos/sanchezviolencia-01.pdf

8. Wolf R, Daichman L, Bennett G. El maltrato de las personas mayores. En: Krug E, Dahlberg L, Mercy J, Zwi A, Lozano R (eds.). World Report on Violence and Health. Washington: World Health Organization, 2003.

9. XLI Asamblea Médica Mundial. Declaración de Hong Kong de la Asociación Médica Mundial sobre el Maltrato a Ancianos. Hong Kong, septiembre de 1989.

10. Ley Orgánica 6/1985 de 1 de julio del Poder Judicial. Artículo 479.

11. Ley Orgánica $1 / 2004$ de 28 de diciembre de Medidas de Protección Integral contra la Violencia de Género. BOE núm. 313 de 29 de diciembre de 2004. p. 42166-97.

12. O'Keeffe M, Hills A, Doyle M, McCreadie C, Scholes S, Constantine R, et al. UK Study of Abuse and Neglect of Older People. Prevalence Survey Report, London 2007, National Centre for Social Research and King's College London.

13. Acierno R, Hernandez MA, Amstadter AB, Resnick HS, Steve K, Muzzy W, et al. Prevalence and correlates of emotional, physical, sexual, and financial abuse and potential neglect in the United States: the National Elder Mistreatment Study. Am J Public Health. 2010 Feb;100(2):292-7. Epub 2009 Dec 17.

14. Vida S, Monks RC, DesRosiers P. Prevalence and correlates of elder abuse and neglect in a geriatric psychiatry service. Can J Psychiatry 2002;47(5):459-67.
15. Cooney C, Howard R, Lawlor B. Abuse of vulnerable people with dementia by their carers: Can we identify those at risk? Int J Geriatric Psychiatry 2006;21: 564-71.

16. CAPV. 2011. Estudio de prevalencia de malos tratos a personas mayores en la Comunidad Autónoma del Pais Vasco. Documentos de Bienestar Social. Departamento de Empleo y Asuntos Sociales. Servicio Central de Publicaciones del Gobierno Vasco. Vitoria-Gazteiz 2011.

17. Bazo MT. Negligencia y malos tratos a las personas ancianas en España. Revista Española de Geriatría y Gerontología 2001;36(1):8-14.

18. Oh J, Kim HS, Martins D, Kim H. Int J Nursing Studies 2006;43:203-14.

19. Tang C, Yan E. Prevalence and Psychological Impact of Chinese Elder Abuse, J Interper Violence 2001;16(11):1158-74.

20. Yan E, Tang C. Elder Abuse by Caregivers: A Study of Prevalence and Risk Factors in Hong Kong Chinese Families. J Family Violence 2004;19(5):269-77.

21. Gomez R, Licely A, Rodríguez G, Krug E. Prevalence of familial elder abuse and associated factors in Ocozocoautla (Chiapas, Mexico). Rev Esp Geriatr Gerontol 2007; 42(1):27-34.

22. Gaioli CC, Rodrigues RA. Occurrence of domestic elder abuse. Rev Lat Am Enfermagem. 2008. MayJun;16(3):465-70.

23. Emlet CA. In-Home Assessment of Older Adults: An Interdisciplinary Approach 2007, 2nd ed. Austin, Pro-Ed.

24. Iborra I. Malos tratos de personas mayores en la familia en España. Fundación de la Comunitat Valenciana para el estudio de la violencia (C.Reina Sofía), 2008.

25. Pérez-Rojo G, Izal M. Marco conceptual. En Instituto de Mayores y Servicios Sociales (IMSERSO), Sociedad Española de Geriatría y Gerontología (SEGG) y Organización Mundial de la Salud (OMS). Malos tratos a personas mayores. Aportación española a los avances internacionales en la adaptación lingüística y cultural de un instrumento de detección de sospecha de malos tratos hacia personas mayores. Ministerio de Trabajo y Asuntos Sociales. 2007.

26. Pérez-Rojo G, Izal M, Montorio I. Nuevo R. Identificación de factores de riesgo de malos tratos hacia personas mayores en el ámbito comunitario. Int J Clinic Health Psychology 2008;8(1):105-17.

27. Risco C, Paniagua MC, Jiménez G, Poblador MD, Molina L, Buitrago $F$. Prevalencia y factores de riesgo de sospecha de malos tratos en la población anciana. Medicina clínica 2005;125(2):51-5.

28. Ruiz A, Altet J, Porta N, Duaso P, Coma M, Requesens $\mathrm{N}$. Violencia doméstica: prevalencia de sospecha de malos tratos a ancianos. Atención Primaria 2001;27:331-4. 